ÉTUDE COMPARATIVE A PRIORI DE DEUX RYTHMES DE REPRODUCTION CHEZ LE LAPIN

\title{
G. MATHERON
}

\author{
Station d'Améliovation Génétique des Animaux \\ IN.R.A., Toulouse, \\ B.P. I2, згз2o Castanet-Tolosan, France
}

Un paramétrage de l'intervalle entre mises-bas en rythme de reproduction post parium et en rythme Io jours chez le lapin nous permet de donner une condition nécessaire et suffisante pour qu'un élevage ro jours gagne à passer en rythme post partum. Pour cela il faut et il suffit que le taux d'abaissement d'une portée suite à une saillie post partum soit inférieur à la part de temps où une femelle est vide entre deux mises-bas dans un rythme semi-intensif. Des tableaux indiquent les gains qui peuverit être réalisés en intensifiant le rythme lorsque la condition est remplie. Le gain exprimé en pourcentage de la prođuctivité numérique annuelle en élevage ro jours dépend du taux de saillies post partum fécondes, du taux de diminution de la taille de la portée lors d'une mise bas post partum et de la part de temps où une femelle est vide entre deux mises-bas en rythme semi-intensif.

ÉTUDE COMPARÉE DE DEUX RYTHMES DE REPRODUCTION CHEZ LE LAPIN DE CHAIR

P. SURDEAU, G. MATHERON, G. PERRIER

Chaive de Productions Animales, E.N.I.T.A. de Dijon, 2I80o Quetigny, France

Quatre-vingt mères issues de deux souches croisées ont été réparties en deux lots homo gènes. Deux unités égales par lot sont soumises l'une à un rythme de saillies le jour de la mise bas, l'autre à des saillies Io jours après la mise bas. L'expérimentation a duré 21 mois (I977-I978).

I'intervalle entre mises bas reste à l'avantage du post partum $(40,35$ jours contre 49,37$)$. Par contre, la prolificité des lapines en rythme semi-intensif est supérieure $(7,8 \mathrm{I}-6,9 \mathrm{I})$. La viabilité des lapereaux dépendant des deux modes de reproduction est semblable.

En définitive, les performances révèlent une productivité numérique analogue. Seuls l'indice de consommation et le taux de réforme sont plus élevés en rythme post partum.

Certains résultats zootechniques apparaissent cependant différenciés avec chaque type génétique contrôlé.

EINFLUSS DES ALTERS UND DES THIOAMINOSÄURENGEHAITS IM FUTTER AUF DIE REPRODUKTIONSLEISTUNG DES KANINCHENS BEI POSTPARTALER INSEMINATION

\author{
W. SCHLOLAUT $(*)$, K. LANGE $\left(^{*}\right)$ und S. PAUFLER (**)
}

(*) Hessische Landesanstalt für Leinstungsprüfungen in der Tievzucht, Neu-Ulrichstain

${ }^{* *}$ Tierärztliches Institut der Universität Göttingen

Es wurde der Einfluß eines durch Zusatz von 0,35 p. 100 DL-Methionin von 0,42 p. Ioo (Gruppe I) auf 0,77 p. I oo (Gruppe 2) erhöhten Thioaminosäurengehaltes im Häsinnen-Alleinfutter bei 326 (Gruppe I) bzw. 3 I 8 (Gruppe 2) Inseminationen in I 8 aufeinanderfolgenden Inseminations-folgen mit insgesamt $4 \mathrm{I}$ Neuseeländer-Häsinnen (weiß) untersucht. Die Insemination erfolgte I-2 Tage post partum entsprechend einem Besamungsintervall von 33 Tagen. Die Erhöhung des Thioaminosäurenanteiles beeinflußte insbesondere die Konseption, welche 68,5 p. I00 in Gruppe I gegenüber 74,5 p. Ioo in Gruppe 2 betrug. Die Differenz war nicht signifikant. Die Wurfstäerke, die Zahl der aufgezogenen Jungtiere, das Gewicht der Jungtiere beim Abstzen, sowie das Gewicht der Häsinnen und deren Futteraufnahme stimmten in beiden Gruppen weit- 